\title{
Comprehensive genotype-phenotype correlation in AP-4 deficiency syndrome; Adding data from a large cohort of Iranian patients
}

\author{
Maryam Beheshtian $^{1}$ | Tara Akhtarkhavari $^{1}$ | Sepideh Mehvari ${ }^{1}$ | \\ Marzieh Mohseni $^{1}$ ( ) | Zohreh Fattahi $^{1}$ | Seyedeh Sedigheh Abedini ${ }^{1}$ | \\ Sanaz Arzhangi $^{1}$ | Mahsa Fadaee ${ }^{1}$ | Payman Jamali ${ }^{2}$ | Reza Najafipour ${ }^{3}$ \\ Vera M. Kalscheuer ${ }^{4}$ () | Hao Hu | Hans-Hilger Ropers $^{4,6}$ (c) | \\ Hossein Najmabadi ${ }^{1,7}$ (1) | Kimia Kahrizi ${ }^{1}$ (c)
}

${ }^{1}$ Genetics Research Center, University of Social Welfare and Rehabilitation Sciences, Tehran, Iran

${ }^{2}$ Genetic Counseling Center, Shahroud Welfare Organization, Semnan, Iran

${ }^{3}$ Cellular and Molecular Research Centre, Genetic Department, Qazvin University of Medical Sciences, Qazvin, Iran

${ }^{4}$ Research Group Development and Disease, Max Planck Institute for Molecular Genetics, Berlin, Germany

${ }^{5}$ Guangzhou Women and Children's Medical Center, Guangzhou, China

${ }^{6}$ Institute for Human Genetics, University Medicine, Mainz, Germany

${ }^{7}$ Kariminejad - Najmabadi Pathology and Genetics Center, Molecular division, Tehran, Islamic Republic of Iran, Tehran, Iran

\section{Correspondence}

Hossein Najmabadi and Kimia Kahrizi, Genetics Research Center, University of Social Welfare and Rehabilitation Sciences, Koodakyar Alley, Daneshjoo Blvd., Evin Street, Tehran, Iran.

Email: hnajm12@yahoo.com (H. N.) and kahrizi@yahoo.com (K. K.)

Funding information Iran National Science Foundation (INSF), Grant/Award Numbers: 950022, 96.11200; National Institute for Medical Research Development (NIMAD), Grant/Award Number: 957060; University of Social Welfare and Rehabilitation Sciences (USWR), Grant/Award Number: 94/801/T/29490

\begin{abstract}
Mutations in adaptor protein complex-4 (AP-4) genes have first been identified in 2009, causing a phenotype termed as AP-4 deficiency syndrome. Since then several patients with overlapping phenotypes, comprised of intellectual disability (ID) and spastic tetraplegia have been reported. To delineate the genotype-phenotype correlation of the AP- 4 deficiency syndrome, we add the data from 30 affected individuals from 12 out of 640 Iranian families with ID in whom we detected disease-causing variants in AP-4 complex subunits, using next-generation sequencing. Furthermore, by comparing genotype-phenotype findings of those affected individuals with previously reported patients, we further refine the genotype-phenotype correlation in this syndrome. The most frequent reported clinical findings in the 101 cases consist of ID and/or global developmental delay (97\%), speech disorders (92.1\%), inability to walk (90.1\%), spasticity $(77.2 \%)$, and microcephaly $(75.2 \%)$. Spastic tetraplegia has been reported in $72.3 \%$ of the investigated patients. The major brain imaging findings are abnormal corpus callosum morphology (63.4\%) followed by ventriculomegaly (44.5\%). Our result might suggest the AP-4 deficiency syndrome as a major differential diagnostic for unknown hereditary neurodegenerative disorders.
\end{abstract}

\section{KEYWORDS}

AP-4 deficiency syndrome, consanguinity, genotype-phenotype correlation, intellectual disability, Iranian families 


\section{1 | INTRODUCTION}

The adaptor protein complex-4 (AP-4) complex, one of the five various adaptor protein complexes, ${ }^{1}$ is localized on the Trans-Golgi Network (TGN), involved in the transport of cargoes from the TGN to endosomes $^{2}$ and autophagosomal structures. ${ }^{3}$ This complex consists of two large chains ( $\beta-4$ and $\varepsilon-4)$, a medium-chain ( $\mu-4)$ and a small chain ( $\sigma-4)$, which are encoded by AP4B1, AP4E1, AP4M1 and AP4S1, respectively. ${ }^{4}$ Supporting evidence has shown that the AP-4 complex contributes to normal development and function of the brain. ${ }^{2}$ Variations in genes encoding these complexes, were first reported by Verkerk et al in 2009, ${ }^{5}$ and then described as a distinct syndrome, AP-4 deficiency syndrome, by Moreno-De-Luca et al in $2011 .^{6}$ This syndrome has been characterized as a subset of hereditary spastic paraplegia, which is very heterogeneous and mainly caused by neurological dysfunctions, such as ID and corpus callosum abnormalities. $5,7,8$

Here we present our findings on 30 affected individuals from 12 Iranian families with disease-causing variants in various subunits of AP-4 complex genes. In order to further delineate the genotypephenotype correlation of the AP-4 deficiency syndrome, we also compared our clinical and molecular findings with previously reported patients in the literature published till mid-March 2020

\section{I MATERIALS AND METHODS}

We selected 12 families from our large cohort of ID patients, in whom disease-causing variants in various subunits of the AP-4 complex have been identified. Our cohort consists of 640 Iranian families, mostly consanguineous with $\geq 2$ affected individuals, who were primarily referred by physicians or clinical geneticists from all over the country to the Genetics Research Center (GRC), between 2007 and 2018 for ID molecular diagnosis. This study was approved by the Ethics Committee at the University of Social Welfare and Rehabilitation Sciences (USWR), Tehran, Iran, and written informed consent was obtained from the legal guardian of patients (Methods in Appendix S1).

\section{3 | RESULTS}

We identified nine different disease-causing variants in AP4M1, AP4B1, AP4S1 and AP4E1 in 50\%, 25\%, $16.7 \%$ and $8.3 \%$ of our 12 Iranian families, respectively (Table S1).

The majority of those families had reported consanguineous marriage (11/12); although one family had a nonconsanguineous condition, both parents had come from the same village (Figure S2-A and Table S1). All disease-causing variants in AP-4 complex subunits were inherited in a homozygous status. Eighteen out of the 30 patients (60\%) were males and the average age of the affected individuals at the last examination was 16.5-year. The age of onset was at early infancy except for three patients, which manifested later in infancy, varied from 2 to 3-year (M-254) (Table S1).
Short stature, ranging from $-2.6 \mathrm{SD}$ to $-7.3 \mathrm{SD}$, and microcephaly, varying from $-2.1 \mathrm{SD}$ to $-7.1 \mathrm{SD}$ were noticed in $86.7 \%$ and $90 \%$ of the patients, respectively. Developmental disability (DD) or ID were reported in all patients; of whom $96.7 \%$ were severely intellectually disabled. Severely impaired speech was noted in all patients. Seizures were reported in $86.7 \%$ of the patients, with seizure manifestation in all patients harboring a variant in AP4B1 or AP4S1. Although all of the patients with disease-causing variants in AP4M1, AP4B1 and AP4S1 developed infantile hypotonia, this phenotype was absent in patients with the AP4E1 variant. All patients manifested spastic tetraplegia and all had lost the ability to walk throughout their life. Spasticity and hypertonia were also universal presentations in all patients. Dysmorphic features were noted in all patients; of which bitemporal narrowing and broad nasal ridge were reported as the most common features. Noteworthy, strabismus was universally observed in the patients. Brain imaging findings of the patients are presented (in supplement), if available. Enlarged sylvian cistern, thin corpus callosum, and ventriculomegaly were reported with higher frequency than other findings in brain images.

Clinical descriptions of the patients, photographs, pedigrees and MRIs are shown in Tables S1 and S2 and Figure S2A,B,C.

Seven patients from three unrelated Iranian families (M-004, M8800140 and $M-8800050)$, living in different geographic locations carry the recurrent AP4M1 variant p.(Glu193Lys). Our previous investigation showed that this variant is located in the haplotype region with a size of $\sim 2.6 \mathrm{Mb}$ shared in the affected individuals (Table S1). ${ }^{9}$

The AP4B1 variant, p.(Thr387Argfs*30) identified in family $M$ 8900295 is also recurrent as it has previously been reported by Abdollahpour et al $(2015)^{10}$ and Szczałuba et al (2020) ${ }^{11}$ in nonconsanguineous families diagnosed with ID and spastic tetraplegia and hereditary spastic paraplegia type 47 (SPG47), respectively (Table S1).

We also report five cases from two unrelated Iranian consanguineous families with ID, carrying a homozygous stop-gain variant, $p$. (Arg97*), in AP4S1. This variant with another frameshift variant, $\mathrm{p}$. (GIn46Profs*9), in trans status has been reported by Hardies et al, ${ }^{12}$ causing mild fever-sensitive seizures, DD and spastic paraplegia in a Caucasian nonconsanguineous family (Table S1).

Iranian patients were quite different from cases in the literature, particularly in terms of facial hypotonia, ability to walk and brain abnormalities (Tables S1 and S2). We summarized all clinic-radiological data of 101 cases in Table 1A-F.

Overall, short stature, abnormality of the face, obesity, stereotypic laughter, inability to walk, and cerebellar and cerebral abnormalities were seen more frequently in patients with AP4E1 disease-causing variants than in patients with variants in other AP-4 complex subunits. In cases with AP4M1 variants, abnormalities of the musculature, limbs and joint were more frequently noticed than in patients with variants in other AP-4 complex subunits. Speech disorder and upper motor neuron dysfunction appeared in all patients with AP4M1 or AP4E1 variants. Furthermore, inability to walk was also more frequently documented in these cases. Aggressive behavior was not reported in patients with the AP4E1 variant; however, stereotypic 
TAB LE 1 Categorizations of clinic-radiological data of 101 cases (including unavailable data) with AP-4 complex subunits in percentage

\begin{tabular}{|c|c|c|c|c|}
\hline Phenotype category & AP4M1 & AP4B1 & AP4S1 & AP4E1 \\
\hline A & \multicolumn{4}{|c|}{ Abnormality of head and growth } \\
\hline Short stature & 45.4 & 50 & 60 & 66.7 \\
\hline Microcephaly & 75.7 & 72.7 & 80 & 77.8 \\
\hline Abnormality of the face & 81.8 & 61.4 & 86.7 & 100 \\
\hline Strabismus & 60.6 & 25 & 53.3 & 33.3 \\
\hline Obesity & 0 & 4.5 & 0 & 33.3 \\
\hline B & \multicolumn{4}{|c|}{ Central nervous system features ${ }^{a}$} \\
\hline Neurodevelopmental delay & 100 & 93.2 & 100 & 100 \\
\hline Seizure & 69.7 & 79.5 & 60 & 55.5 \\
\hline Speech disorder & 100 & 86.4 & 86.7 & 100 \\
\hline Inability to walk & 97 & 84.1 & 86.7 & 100 \\
\hline Upper motor neuron dysfunction & 100 & 79.5 & 93.3 & 100 \\
\hline Abnormal reflex & 75.7 & 70.4 & 60 & 55.5 \\
\hline Pseudobulbar sign & 30.3 & 4.5 & 0 & 22.2 \\
\hline Drooling & 54.5 & 25 & 53.3 & 44.4 \\
\hline C & \multicolumn{4}{|c|}{ Abnormality of the musculature ${ }^{b}$} \\
\hline Abnormality of the musculature & 100 & 75 & 73.3 & 66.7 \\
\hline Abnormality of limbs & 69.7 & 40.9 & 53.3 & 22.2 \\
\hline Abnormality of joint mobility & 63.6 & 40.9 & 53.3 & 55.5 \\
\hline $\mathrm{D}$ & \multicolumn{4}{|c|}{ Behavioral abnormalities } \\
\hline Aggressive behavior & 18.2 & 6.8 & 0 & 0 \\
\hline Shyness & 21.2 & 11.4 & 33.3 & 22.2 \\
\hline Stereotypic laughter & 63.6 & 34.1 & 53.3 & 88.9 \\
\hline $\mathrm{E}$ & \multicolumn{4}{|c|}{ Brain MRI abnormalities } \\
\hline Ventriculomegaly & 66.7 & 34.1 & 20 & 55.5 \\
\hline Hypoplasia of the corpus callosum & 66.7 & 72.7 & 40 & 44.4 \\
\hline White matter abnormalities & 24.2 & 40.9 & 26.7 & 11.1 \\
\hline Enlarged sylvian cistern & 36.4 & 20.4 & 33.3 & 44.4 \\
\hline Abnormal morphology of the hippocampus & 42.4 & 11.4 & 0 & 44.4 \\
\hline Abnormality of the pons & 9.1 & 2.3 & 0 & 0 \\
\hline Gliosis & 3 & 0 & 0 & 0 \\
\hline Cerebral degeneration & 3 & 11.4 & 0 & 33.3 \\
\hline Aplasia/Hypoplasia of the cerebellum & 33.3 & 2.3 & 0 & 44.4 \\
\hline $\mathrm{F}$ & \multicolumn{4}{|c|}{ Facial dysmorphisms in details } \\
\hline Coarse facial features & 33.3 & 2.3 & 20 & 44.4 \\
\hline Long \& narrow face & 0 & 6.8 & 0 & 22.2 \\
\hline Bitemporal narrowing & 45.4 & 22.7 & 33.3 & 55.5 \\
\hline Broad nasal ridge & 48.5 & 29.5 & 33.3 & 55.5 \\
\hline Bulbous nose & 51.5 & 27.3 & 66.7 & 44.4 \\
\hline Long nose & 0 & 0 & 0 & 22.2 \\
\hline Broad nasal bridge & 36.4 & 25.0 & 46.7 & 33.3 \\
\hline Prominent nose & 18.2 & 4.5 & 40 & 44.4 \\
\hline Short philtrum & 33.3 & 15.9 & 46.7 & 22.2 \\
\hline Everted upper lip vermilion & 45.4 & 22.7 & 33.3 & 22.2 \\
\hline Full lips & 0 & 0 & 13.3 & 0 \\
\hline Wide mouth & 57.6 & 22.7 & 66.7 & 44.4 \\
\hline
\end{tabular}


TABLE 1 (Continued)

\begin{tabular}{|lllll}
\hline Phenotype category & AP4M1 & AP4B1 & AP4S1 & AP4E1 \\
\hline High palate & 33.3 & 22.7 & 46.7 & 0 \\
\hline Hypertelorism & 0 & 2.3 & 13.3 & 20 \\
\hline Pointed chin & 0 & 0 & 20 & 22.2 \\
\hline Prominent antihelix & 9.1 & 11.4 & 0 & 0 \\
\hline Downslanted palpebral fissures & 0 & 0 & 22.2
\end{tabular}

Abbreviation: AP-4, adaptor protein complex-4.

${ }^{a}$ Neurodevelopmental abnormality indicates for global developmental delay and intellectual disability; Upper motor neuron dysfunction indicates for spastic tetraplegia/tetraparesis/paraplegia, hypertonia and spasticity; Abnormal reflex includes hyperreflexia and positive Babinski sign.

${ }^{\mathrm{b}} \mathrm{Abnormality}$ of the musculature includes infantile muscular hypotonia, low or weak muscle tone, decreased muscle mass and abnormality of facial musculature; Abnormality of the limbs includes talipes equinovarus, pes planus and adducted thumb; Joint mobility abnormalities include contractures and joint laxity.

laughter was more frequent in these cases than in cases with variants in other AP4-subunits. Although ventriculomegaly was as prevalent as corpus callosum abnormality in patients with AP4M1 variants, for those with AP4E1 alterations, ventriculomegaly was documented more frequently than the corpus callosum abnormality. In cases with AP4B1 or AP4S1 variants, abnormality of the corpus callosum was more common reported than ventriculomegaly. Cerebellar and cerebral abnormalities were not present in any case with AP4S1 variation.

Moreover, through data investigation in the pfam database (https://pfam.xfam.org/), we depicted the location of each identified disease-causing variant in the corresponding AP-4 complex subunits, in the schematic illustration of domain structures in Figure $S 1$.

Totally, we combined 12, 23, 6 and 4 variants in AP4M1, AP4B1, AP4S1 and AP4E1, respectively.

\section{DISCUSSION}

Since the AP-4 deficiency syndrome was first reported by Verkerk et $\mathrm{al}^{5}$ in 2009, and homozygous AP4M1 variants were identified, causing ID and tetraplegic cerebral palsy (SPG50; OMIM\# 612936), many other patients with overlapping clinical phenotypes have been reported, in whom the diseases resulted from pathogenic variants in AP-4 subunits such as AP4E1 (SPG51; OMIM\#613744), AP4B1 (SPG47; OMIM\#614066), and AP4S1 (SPG52; OMIM\#614067)..$^{6,8,12-14}$ Here, we discuss the phenotype-genotype correlation of 101 patients in the spectrum of AP-4 related diseases.

The highest number of inherited disease-causing variants among various subunits of the AP-4 complex in Iranian cases belongs to $A P 4 M 1$, followed by AP4B1, AP4S1 and AP4E1, respectively; however, combining our data with data from non-Iranian patients places AP4B1 at the top. Eight out of 44 cases (18.2\%) with the AP4B1 variants were from Iran (Tables S1 and S2).

Thirty-two out of the 57 families (56.1\%) discussed in this paper, reported consanguineous marriages; of which $34.4 \%$ were Iranian. Biallelic variants in homozygous state were the most common alterations in AP-4 deficiency syndrome (Table S1 and Figure S1).

\section{1 | Major clinical findings in AP-4 deficiency syndrome}

Our findings and those reported by others combined suggest that the major clinical findings with nearly two-third prevalence in AP-4 deficiency are as follows (Table 1A-D and Table S2):

Global developmental delay and/or ID (97\%); speech disorder (92.1\%); inability to walk (90.1\%); spasticity (77.2\%); microcephaly (75.2\%); spastic tetraplegia and infantile muscular hypotonia (72.3\%); seizures (71.3\%); hyperreflexia (63.4\%) and hypertonia (62.4\%).

In line with previous studies, which concluded that seizure is a variable manifestation in AP-4 deficiency syndrome, ${ }^{12,15} 28.7 \%$ of the cases described here did not manifest seizures.

Although spastic tetraplegia and spasticity were not reported in $27.7 \%$ and $22.8 \%$ of all cases, respectively, hypotonic developmental delay might serve as the initial manifestation of tetraplegia and spasticity in patients with AP4 variants, as suggested before. ${ }^{5,6,8,15}$

Nearly $10 \%$ of all cases described here were able to walk in the time of the report. However, it is probable that this ability will regress over time because of spasticity and/or foot deformities. ${ }^{5,6,8,15}$

Dysmorphic facial features were reported in 76 out of 101 cases. Bulbous nose and wide mouth were the most frequent facial abnormalities (42.6\%) (Table 1F \& Table S1).

In 82 out of 101 cases at least one brain abnormality was reported. Major characteristic findings in brain MRIs comprised abnormal corpus callosum morphology (63.4\%), ventriculomegaly (44.5\%), enlarged cisterns and white matter abnormalities ( 30\%) (Table 1E \& Tables S1 and S2).

\section{2 | Additional clinical findings in AP-4 deficiency syndrome}

There are some extra clinical features manifested at least in $25 \%$ of the patients. These included short stature (51.5\%), drooling (40.6\%), low or weak muscle tone (32.7\%), talipes equinovarus (39.6\%), contractures (48.5\%), and Babinski sign (53.5\%) (Table S2). 
In psychiatric behavior, stereotypic laughter was the most common feature that appeared in $51.5 \%$ of the cases.

Table S3 presents other less frequent findings in these patients. These features might be simply a coincidental findings and not the straight result of AP-4 deficiency.

\subsection{More heterogeneity in patients harboring variations in AP-4 complex subunits}

In a Moroccan consanguineous family with neurodegeneration associated with brain iron accumulation, a disease-causing AP4M1 variant (p.Arg306*) has been reported. ${ }^{16}$ Like other cases with AP4M1 mutations, the neurological findings were common in these patients. In addition to ventriculomegaly, thinning of the splenium of the corpus callosum, cerebral atrophy and white matter loss, they had also marked bilateral pallidal iron accumulation on brain MRI of their cases (Tables S1 \& 3). ${ }^{16}$

Also, we included a homozygous AP4S1 variant, c.138 +3_6delAAGT, which has been reported in two unrelated families from Albania and Kosovo with totally three cases (Table S1). ${ }^{17,18}$ Those differ in some features in terms of facial abnormalities, spasticity, and seizures. Vill et al reported some additional clinical features in their patient, such as bilateral hypointense signal changes in the globus pallidus in favor of brain iron or copper accumulation, and ataxia. $^{18}$

However, we did not observe any evidence suggestive of brain iron accumulation in Iranian patients even in the oldest one (32-year). Moreover, Behne et al in 2020 showed unchanged iron content in the fibroblasts derived from patients with AP-4 deficiency syndrome, ${ }^{19}$ which might be indicative of further investigation.

In conclusion, our findings add more evidence to the growing body of genotype-phenotype data in patients with diseasecausing variants in AP-4 complex subunits. Furthermore, identification of disease-causing variants in AP-4 complex subunits in 12 out $640(\sim 2 \%)$ Iranian families with inherited ID, might reflect the high prevalence of AP-4 Deficiency Syndrome among Iranian ID patients.

\section{ACKNOWLEDGEMENT}

We thank all families for their contribution. This study was supported by the Iran National Science Foundation (INSF) with grant no. 96.11200 to K. K. and grant no. 950022 to H. N., and National Institute for Medical Research Development (NIMAD) with grant no. 957060 to K. K., and University of Social Welfare and Rehabilitation Sciences (USWR) grant no.94/801/T/29490 to H. N.

\section{CONFLICT OF INTEREST}

Nothing to declare.

\section{PEER REVIEW}

The peer review history for this article is available at https://publons. com/publon/10.1111/cge.13845.

\section{DATA AVAILABILITY STATEMENT}

The data supporting our findings in Iranian patients are openly available at doi: 10.1038/nature10423; doi: 10.1038/s41380-0170012-2; and doi: 10.1111/cge.13463. Data sharing is not applicable as no new data were created in this study.

\section{ORCID}

Marzieh Mohseni (D) https://orcid.org/0000-0003-1200-4044 Vera M. Kalscheuer (D) https://orcid.org/0000-0001-6898-3259 Hans-Hilger Ropers (D) https://orcid.org/0000-0002-8552-9813 Hossein Najmabadi (D) https://orcid.org/0000-0002-6084-7778 Kimia Kahrizi (D) https://orcid.org/0000-0002-6587-7706

\section{REFERENCES}

1. Park SY, Guo X. Adaptor protein complexes and intracellular transport. Biosci Rep. 2014;34(4):381-390.

2. Hirst J, Irving C, Borner GH. Adaptor protein complexes AP-4 and AP-5: new players in endosomal trafficking and progressive spastic paraplegia. Traffic. 2013;14(2):153-164.

3. Matsuda S, Miura E, Matsuda K, et al. Accumulation of AMPA receptors in autophagosomes in neuronal axons lacking adaptor protein AP-4. Neuron. 2008;57(5):730-745.

4. Matsuda S, Yuzaki M. Polarized sorting of AMPA receptors to the somatodendritic domain is regulated by adaptor protein AP-4. Neurosci Res. 2009;65(1):1-5.

5. Verkerk AJ, Schot R, Dumee B, et al. Mutation in the AP4M1 gene provides a model for neuroaxonal injury in cerebral palsy. Am J Hum Genet. 2009;85(1):40-52.

6. Moreno-De-Luca A, Helmers SL, Mao H, et al. Adaptor protein complex-4 (AP-4) deficiency causes a novel autosomal recessive cerebral palsy syndrome with microcephaly and intellectual disability. J Med Genet. 2011;48(2):141-144.

7. Guardia CM, De Pace R, Mattera R, Bonifacino JS. Neuronal functions of adaptor complexes involved in protein sorting. Curr Opin Neurobiol. 2018;51:103-110.

8. Abou Jamra R, Philippe O, Raas-Rothschild A, et al. Adaptor protein complex 4 deficiency causes severe autosomal-recessive intellectual disability, progressive spastic paraplegia, shy character, and short stature. Am J Hum Genet. 2011;88(6):788-795.

9. $\mathrm{Hu} \mathrm{H}$, Kahrizi K, Musante L, et al. Genetics of intellectual disability in consanguineous families. Mol Psychiatry. 2019;24(7):1027-1039.

10. Abdollahpour $\mathrm{H}$, Alawi $\mathrm{M}$, Kortum $\mathrm{F}$, et al. An AP4B1 frameshift mutation in siblings with intellectual disability and spastic tetraplegia further delineates the AP-4 deficiency syndrome. Eur J Hum Genet. 2015;23(2):256-259.

11. Szczaluba K, Mierzewska H, Smigiel R, et al. AP4B1-associated hereditary spastic paraplegia: expansion of phenotypic spectrum related to homozygous p.Thr387fs variant. J Appl Genet. 2020;61(2):213-218.

12. Hardies K, May P, Djemie T, et al. Recessive loss-of-function mutations in AP4S1 cause mild fever-sensitive seizures, developmental delay and spastic paraplegia through loss of AP-4 complex assembly. Hum Mol Genet. 2015;24(8):2218-2227.

13. Bauer $P$, Leshinsky-Silver E, Blumkin L, et al. Mutation in the AP4B1 gene cause hereditary spastic paraplegia type 47 (SPG47). Neurogenetics. 2012;13(1):73-76.

14. Blumkin L, Lerman-Sagie T, Lev D, Yosovich K, Leshinsky-Silver E. A new locus (SPG47) maps to 1p13.2-1p12 in an Arabic family with complicated autosomal recessive hereditary spastic paraplegia and thin corpus callosum. J Neurol Sci. 2011;305(1-2):67-70.

15. Tuysuz B, Bilguvar $\mathrm{K}$, Kocer $\mathrm{N}$, et al. Autosomal recessive spastic tetraplegia caused by AP4M1 and AP4B1 gene mutation: expansion 
of the facial and neuroimaging features. Am J Med Genet A. 2014; 164a(7):1677-1685.

16. Roubertie A, Hieu N, Roux CJ, et al. AP4 deficiency: a novel form of neurodegeneration with brain iron accumulation? Neurol Genet. 2018; 4(1):e217.

17. Tessa A, Battini R, Rubegni $A$, et al. Identification of mutations in AP4S1/SPG52 through next generation sequencing in three families. Eur J Neurol. 2016;23(10):1580-1587.

18. Vill K, Muller-Felber W, Alhaddad B, et al. A homozygous splice variant in AP4S1 mimicking neurodegeneration with brain iron accumulation. Mov Disord. 2017;32(5):797-799.

19. Behne R, Teinert J, Wimmer M, et al. Adaptor protein complex 4 deficiency: a paradigm of childhood-onset hereditary spastic paraplegia caused by defective protein trafficking. Hum Mol Genet. 2020;29(2):320-334.

\section{SUPPORTING INFORMATION}

Additional supporting information may be found online in the Supporting Information section at the end of this article.

How to cite this article: Beheshtian M, Akhtarkhavari T, Mehvari S, et al. Comprehensive genotype-phenotype correlation in AP-4 deficiency syndrome; Adding data from a large cohort of Iranian patients. Clinical Genetics. 2020;1-6. https://doi.org/10.1111/cge.13845 